\title{
Hippocampal Volume Reduction in Humans Predicts Impaired Allocentric Spatial Memory in Virtual-Reality Navigation
}

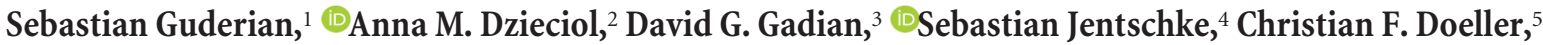 \\ Neil Burgess, ${ }^{6}{ }^{\circ}$ Mortimer Mishkin, ${ }^{1}$ and Faraneh Vargha-Khadem ${ }^{2}$ \\ ${ }^{1}$ Laboratory of Neuropsychology, National Institute of Mental Health, National Institutes of Health, Bethesda, Maryland 20892, ${ }^{2}$ Cognitive Neuroscience \\ and Neuropsychiatry Section, University College London Institute of Child Health, and Great Ormond Street Hospital, London WC1N 1EH, United \\ Kingdom, ${ }^{3}$ Developmental Imaging and Biophysics Section, University College London Institute of Child Health, London WC1N 1EH, United Kingdom, \\ ${ }^{4}$ Cluster "Languages of Emotion," Freie Universität Berlin, Berlin 14195, Germany, ${ }^{5}$ Donders Institute for Brain, Cognition and Behaviour, Radboud \\ University, 6500 HB Nijmegen, The Netherlands, and ${ }^{~ I n s t i t u t e ~ o f ~ C o g n i t i v e ~ N e u r o s c i e n c e ~ a n d ~ I n s t i t u t e ~ o f ~ N e u r o l o g y, ~ U n i v e r s i t y ~ C o l l e g e ~ L o n d o n, ~ L o n d o n ~}$ \\ WC1N 3AR, United Kingdom
}

The extent to which navigational spatial memory depends on hippocampal integrity in humans is not well documented. We investigated allocentric spatial recall using a virtual environment in a group of patients with severe hippocampal damage (SHD), a group of patients with "moderate" hippocampal damage (MHD), and a normal control group. Through four learning blocks with feedback, participants learned the target locations of four different objects in a circular arena. Distal cues were present throughout the experiment to provide orientation. A circular boundary as well as an intra-arena landmark provided spatial reference frames. During a subsequent test phase, recall of all four objects was tested with only the boundary or the landmark being present. Patients with SHD were impaired in both phases of this task. Across groups, performance on both types of spatial recall was highly correlated with memory quotient (MQ), but not with intelligence quotient (IQ), age, or sex. However, both measures of spatial recall separated experimental groups beyond what would be expected based on MQ, a widely used measure of general memory function. Boundary-based and landmark-based spatial recall were both strongly related to bilateral hippocampal volumes, but not to volumes of the thalamus, putamen, pallidum, nucleus accumbens, or caudate nucleus. The results show that boundary-based and landmark-based allocentric spatial recall are similarly impaired in patients with SHD, that both types of recall are impaired beyond that predicted by MQ, and that recall deficits are best explained by a reduction in bilateral hippocampal volumes.

Key words: amnesia; human; memory; navigation; spatial; virtual reality

\section{Significance Statement}

In humans, bilateral hippocampal atrophy can lead to profound impairments in episodic memory. Across species, perhaps the most well-established contribution of the hippocampus to memory is not to episodic memory generally but to allocentric spatial memory. However, the extent to which navigational spatial memory depends on hippocampal integrity in humans is not well documented. We investigated spatial recall using a virtual environment in two groups of patients with hippocampal damage (moderate/severe) and a normal control group. The results showed that patients with severe hippocampal damage are impaired in learning and recalling allocentric spatial information. Furthermore, hippocampal volume reduction impaired allocentric navigation beyond what can be predicted by memory quotient as a widely used measure of general memory function.

\section{Introduction}

In humans, hypoxic/ischemic events early in life can lead to marked bilateral hippocampal atrophy, which has been associated with profound impairments in episodic memory while leav- ing semantic memory relatively intact (Vargha-Khadem et al., 1997), and with marked deficits in recall while largely sparing

This work was supported by Medical Research Council Grant G0300117/65439, Intramural Research Program of the National Institute of Mental Health, National Institutes of Health, Department of Health and Human Services Project ZIAMH000478, UK Central and East London Research Network (5177), the European Research Council (ERC StG 261177) and the Netherlands Organisation for Scientific Research (NWO-Vidi 45212009). We thank all the participants in this study, in particular, the patients and their families. 
recognition memory (Adlam et al., 2009). Across species, however, perhaps the most well-established contribution of the hippocampus to memory is not to episodic memory generally but to allocentric spatial memory. Thus, hippocampal damage has been associated with deficits in allocentric spatial memory in rodents (Morris et al., 1982; Packard and McGaugh, 1996; Pearce et al., 1998) and in nonhuman primates (Hampton et al., 2004; Lavenex et al., 2006; Glavis-Bloom et al., 2013; Forcelli et al., 2014), as well as in humans (Holdstock et al., 2000; Spiers et al., 2001; King et al., 2002, 2004; Hartley et al., 2007; Kumaran et al., 2007). The majority of the studies in experimental animals involve a navigational component (i.e., requiring movement to a remembered location), whereas the human studies have examined primarily spatial relational memory using tests that did not assess navigation. Thus, the extent to which patients with relatively selective hippocampal damage are impaired in allocentric navigational spatial memory is not well documented.

Interestingly, recent evidence suggests that the reliance of allocentric spatial memory ability on hippocampal integrity in humans may not be absolute. In a virtual-reality-based place recall experiment using fMRI in healthy human participants, Doeller et al. (2008) found that, whereas learning and remembering object location relative to local boundaries were associated with hippocampal activity, learning and remembering object location relative to local landmarks were associated instead with activation of the caudate nucleus (Doeller et al., 2008). This finding is consistent with results from rodent experiments (Packard and McGaugh, 1996; Pearce et al., 1998) and suggests that patients with selective hippocampal damage may be able to learn and retrieve location information relative to local landmarks, but not location information relative to environmental boundaries.

To test this possibility, we adapted the allocentric spatial recall paradigm in a virtual environment used by Doeller et al. (2008) to test two groups of patients who had suffered from hypoxia/ischemia early in life - one that had severe hippocampal damage (SHD, $N=15$; volume reduction mean, $46.6 \%$; range, $30.4 \%-$ $66.8 \%$ ), one that had "moderate" hippocampal damage (MHD, $N=13$; volume reduction mean, $19.3 \%$; range, $14.6 \%-$ $25.8 \%)$ - a normal control group $(N=19)$. The virtual environment consisted of a circular arena, with distal cues being present throughout the experiment to provide orientation. Through four blocks of trials with feedback, participants learned the target locations of four different objects (one trial for each object in each block). During learning, a circular boundary, as well as an intraarena landmark provided spatial reference frames. During a subsequent test phase, recall of all four objects was tested with only the circular boundary or the landmark being present. The behavioral measure of interest was the recall error, as measured by the Euclidean distance between the recalled location and the target location for each object. Below, recall errors are termed "boundary error" and "landmark error" for boundary-based and landmark-based navigation, respectively.

The authors declare no competing financial interests.

This article is freely available online through the J Neurosci Author Open Choice option.

Correspondence should be addressed to either of the following: Dr. Sebastian Guderian, Laboratory of Neuropsychology, National Institute of Mental Health, National Institutes of Health, 49 Convent Drive, Room 1B80, Bethesda, MD 20892, E-mail: guderians@mail.nih.gov; or Dr. Faraneh Vargha-Khadem, Section on Cognitive Neuroscience and Neuropsychiatry, University College London, Institute of Child Health, and Great Ormond Street Hospital, 30 Guilford Street, London WC1N 1EH, UK, E-mail: f.vargha-khadem@ucl.ac.uk.

DOI:10.1523/JNEUROSCI.0801-15.2015

Copyright $\odot 2015$ Guderian et al.

This is an Open Access article distributed under the terms of the Creative Commons Attribution License Creative Commons Attribution 4.0 International, which permits unrestricted use, distribution and reproduction in any medium provided that the original work is properly attributed.

\section{Materials and Methods \\ Participants}

The study was approved by the Research Ethics Committee of University College London Hospital. Informed consent was provided by parents and their children and by young adult participants, as appropriate.

A total of 15 patients with SHD ( 6 females; mean \pm SD age: $21.5 \pm 7.7$ years), 13 patients with MHD (6 females; mean \pm SD age: $13.2 \pm 2.4$ years), and 19 controls ( 9 females; mean \pm SD age: $18.9 \pm 8.4$ years) were included in the study. Presence of overt neurological impairment and of genetic syndromes were exclusion criteria. All patients in the MHD and SHD groups suffered from hypoxic/ischemic events either perinatally ( $n=24)$ or in early childhood $(n=4)$. Patients with a history of hypoxia/ ischemia were assigned to the MHD group if the hippocampal volume (corrected for intracranial volume) was reduced by $>10 \%$ and $<30 \%$ (mean, 19.3\%; range, $14.6 \%-25.8 \%$ ), and to the SHD group if the hippocampal volume reduction was $>30 \%$ (mean, $46.6 \%$; range, $30.4 \%$ $66.8 \%$ ) (Isaacs et al., 2000, 2003), relative to the control group (mean, $0 \%$, by definition; range, $-13.5 \%$ to $13.8 \%$ ). The mean hippocampal volumes in cubic millimeters ([left + right $] / 2$; not corrected for intracranial volume) were 3324 (SD 324) for controls, 2590 (SD 282) for patients with MHD, and 1684 (SD 361) for patients with SHD.

\section{Etiology of hypoxic/ischemic events}

The SHD group had the following: transposition of the great arteries and open heart surgery during the neonatal period $(n=2)$; complications associated with prematurity, neonatally/perinatally $(n=3)$; hypoplastic left heart syndrome and Norwood operations, perinatally and later up to age $4(n=1)$; acute hypoxemic respiratory failure as neonates $(n=2)$; hypoglycemic attacks starting at age 9 and culminating in a severe attack at age $15(n=1)$; epilepsy of early onset $(n=2)$; drug toxicity and cardiac arrest at age $9(n=1)$; and acute perinatal encephalopathy $(n=3)$.

The MHD group had the following: acute hypoxemic respiratory failure neonatally $(n=7)$; and transposition of the great arteries and open heart surgery neonatally $(n=6)$.

\section{Assessment of intelligence quotient (IQ) and memory quotient (MQ)}

Full-scale IQs were calculated for each participant using the Wechsler Adult Intelligence Scale, Ed 3 (Wechsler, 1997) or the Wechsler Intelligence Scale for Children, Ed 4 (Wechsler, 2003). These scales provide four Index scores: Verbal Comprehension Index, measuring understanding of verbal concepts; Perceptual Reasoning Index, reflecting nonverbal perception and manipulation; Working Memory Index, tracking on-line memory; and Processing Speed Index, measuring speed of perceptual reasoning on routine visuomotor tasks. The Full Scale IQ and the four Index scores are expressed as standard scores with a mean of 100 and an SD of 15 .

An MQ was calculated for each participant using either the Children's Memory Scale (CMS; Cohen, 1997) or the Wechsler Memory Scale, Ed 3 (for adults). These scales provide measures of immediate and delayed memory for verbal and visual information. Both scales provide standard scores with a mean of 100 and an SD of 15 for General Memory.

Figure 1 shows the distributions of age, IQ, and MQ for the three experimental groups.

\section{Structural imaging acquisition protocols and volumetric measurements}

Whole-brain MRI scans were obtained using a 1.5-T Siemens Avanto scanner, with a T1-weighted 3D FLASH sequence: repetition time, $11 \mathrm{~ms}$; echo time, $4.94 \mathrm{~ms}$; flip angle, $15^{\circ}$; matrix size, $224 \times 256$; field of view, $250 \mathrm{~mm}$; partition thickness, $1 \mathrm{~mm}$; 176 sagittal partitions in the third dimension; acquisition time, $5.34 \mathrm{~min}$.

The thalamus, caudate nucleus, putamen, globus pallidus, and nucleus accumbens were segmented semiautomatically using the FSL FIRST software package (Patenaude et al., 2011) by one of the authors (A.M.D.). Automatic segmentations were inspected for accuracy, and minor errors were corrected manually. The segmentations of the hippocampus, also 

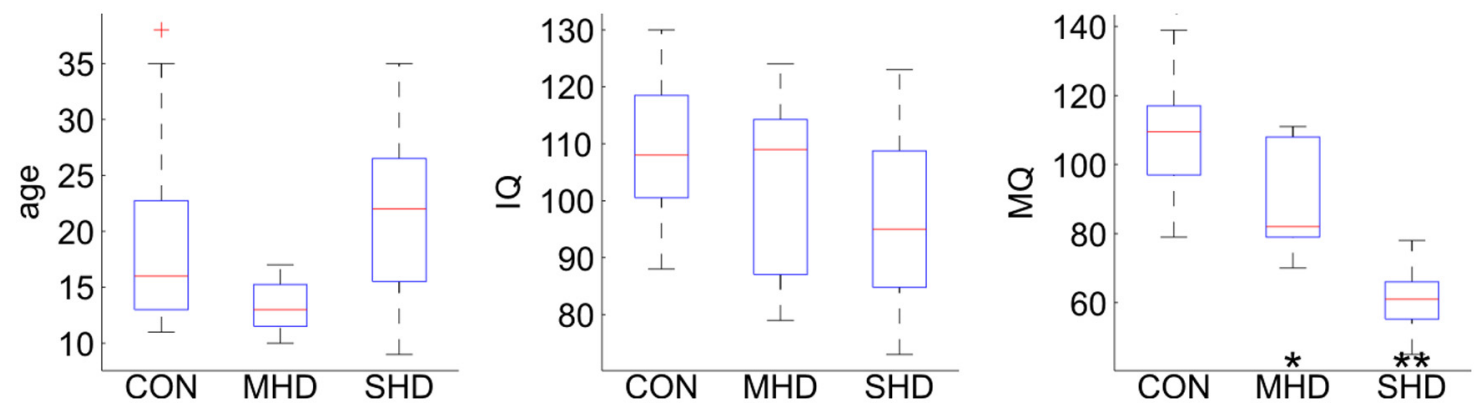

Figure 1. Age, IQ, and MQ for the three experimental groups. On each box: Horizontal red line indicates the median. Edges of the box indicate the 25th (q1) and 75th (q3) percentiles. Whiskers extend to the most extreme data points not considered outliers. Points are considered outliers (displayed as a red +$)$ if they are larger than $q 3+(q 3-q 1)$ or smaller than q1 $-(q 3-q 1)$. ${ }^{*} p<0.01$, patient groups compared with the control group ( $t$ test $) .{ }^{* *} p<0.001$, patient groups compared with the control group ( $t$ test).

Hip

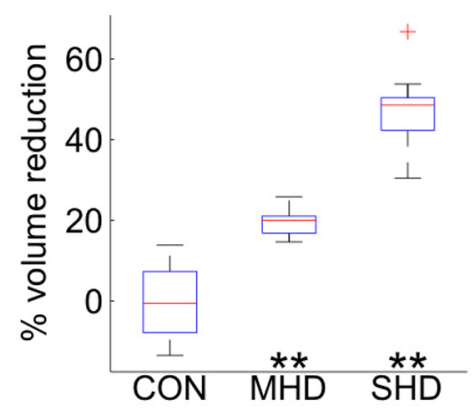

Pall

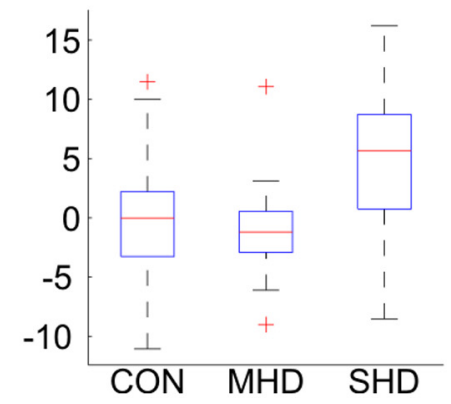

Thal

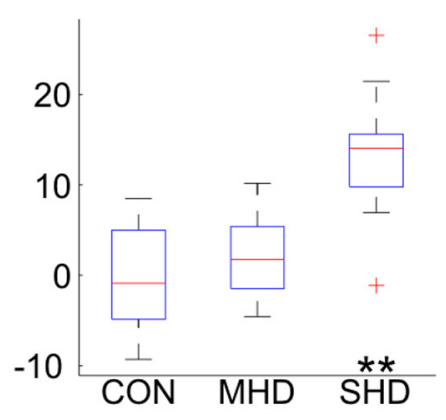

NAcc

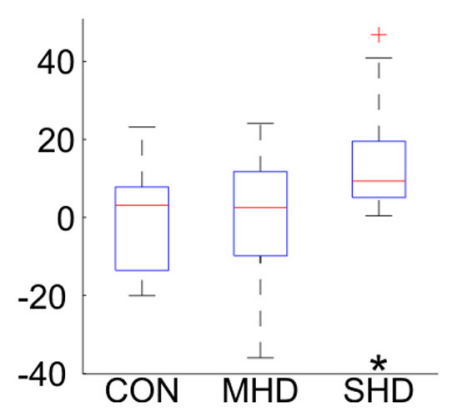

Put

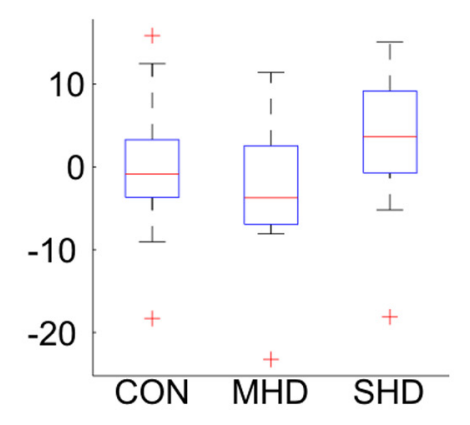

Caud

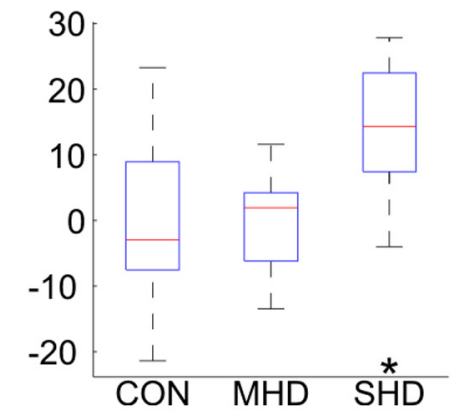

Figure 2. Percentage volume reduction relative to the control group mean. Box properties are the same as in Figure 1. Positive values correspond to volumes smaller than the control group mean. ${ }^{*} p<0.01$, patient groups compared with the control group ( $t$ test). ${ }^{* *} p<0.001$, patient groups compared with the control group ( $t$ test). Hip, Hippocampus; Thal, thalamus; Put, putamen; Pall, pallidum; NAcc, nucleus accumbens; caud, caudate nucleus.

provided by FSL FIRST, were judged to be inaccurate for many patients with SHD due to the changed shape of the atrophied hippocampus. Hippocampal volumes of all participants were therefore measured manually by one of the authors (D.G.G.) according to a published protocol (Cooper et al., 2015).

All structure volumes were corrected for brain size by dividing them by intracranial volumes obtained from the SPM8 "New Segment" protocol. In all analyses, the volumes are therefore expressed as percentages of total intracranial volume.

Figure 2 shows the structure volumes for the three experimental groups, expressed as percentage volume reduction relative to the control group mean.

\section{Spatial recall task}

The virtual environment consisted of a circular arena, with distal cues being present throughout the experiment (learning phase and test phase) to provide orientation. Participants used the forward, left, and right arrow keys on a keyboard to navigate in the environment.
For all participants, the experiment was separated into a learning phase and a test phase.

Throughout the learning phase, a circular boundary as well as an intraarena landmark provided spatial reference frames. Participants were first allowed to familiarize themselves with the manual controls and the environment by navigating around the arena. After this familiarization phase, participants learned the location of four objects (a rubber duck, a vase, a basketball, and a bucket) in 5 blocks. Each block consisted of four trials, one for each object. The target location of these objects in the environment was fixed throughout the experiment.

The first block consisted of 4 trials, one for each object, during which the object was placed at its target location. The task was to "collect the object" at the target location by "walking" over it using the manual controls.

Each one of blocks 2-5 again consisted of four trials, one for each object. In each trial, participants were first cued with one of the objects, displayed centrally on the screen in front of a gray background. Participants started successive trials from a different starting position. The task 
was to navigate to the object's target location and to press the space bar once participants thought they were at the correct location. After pressing the space bar, the object appeared in its correct location, and participants again had to collect the object by walking over it before the next trial. Thus, feedback was provided on each learning trial. Throughout each trial, a small icon of the current target object was shown in the top left corner of the screen.

The test phase consisted of four blocks. In each block, recall of two of the four objects was tested. Four test trials were given in each block, with each object being tested twice, each time from a different starting position to prevent the use of egocentric navigational strategies. In each block, either the circular boundary (B) or the landmark (L) was present in isolation. For approximately half the participants, the order of the blocks was LBBL, and for the other half it was BLLB. Across all four blocks, each object was tested twice in each condition (B and L), for a total of 16 test trials. Just as in blocks $2-5$ of the learning phase, participants were first cued with one of the objects, and a small icon of the current target object was shown in the top left corner of the screen throughout the trial. Participants again were instructed to press the space bar once they thought they were at the correct location.

\section{Data analysis}

The measure of interest for spatial recall accuracy was the distance error (i.e., the distance between an object's target location and the location at which the participant dropped the target object). All analyses were performed using MATLAB (The MathWorks).

\section{Learning data}

Learning of the object locations was assessed by analyzing blocks 2-5 (labeled 1-4 in Fig. 5) of the learning phase. For each participant, the distance error was first averaged across the four trials/object for each block. A mixed effects model with "block" as a continuous predictor, "group" as a fixed factor (three levels: normal controls, MHD patients, and SHD patients), and "subject" as a random factor nested in "group" was used to assess whether performance and learning rate differed among the groups. In addition, post hoc analyses were performed on the three groups separately.

\section{Test data}

Cluster analysis. We first used K-means cluster analysis as a completely data-driven approach, to test whether spatial memory performance in the boundary-only and landmark-only conditions separates the three experimental groups (SHD, MHD, and normal controls). The procedure described by Caliński and Harabasz (1974) was used to assess the number of separable clusters. Each cluster analysis was performed 1000 times with a new random set of initial cluster centroids, to prevent convergence to a local minimum. Three analyses were performed: one on both boundary and landmark errors together, one using only boundary error, and one using only landmark error. Distance errors for the boundary and landmark errors were separately normalized between 0 and 1 across groups $\left[x=\frac{x-\min (x)}{\max (x)-\min (x)}\right]$. This was done because the distance error had an upper bound in the boundary-only condition (the boundary preventing navigation beyond it), whereas there was no upper bound for the distance error in the landmark-only condition (the absence of the boundary removes the constraint on navigation). Normalizing the distance errors for the two conditions separately across groups thus removes any absolute differences between boundary and landmark errors regardless of group while retaining relative differences in distance errors among groups.

Regression analyses. Two multiple regression analyses were performed to assess how the distance errors were related, on the one hand, to behavioral and demographic measures, and on the other, to measures of brain structural volumes. For the regression analysis using the behavioral and demographic measures, the overall MQ, Full Scale IQ, age, and sex were used as predictor variables. For the regression analysis using measures of brain structures, the volumes of the hippocampus, thalamus, putamen, globus pallidus, nucleus accumbens, and caudate nucleus were used as predictor variables.

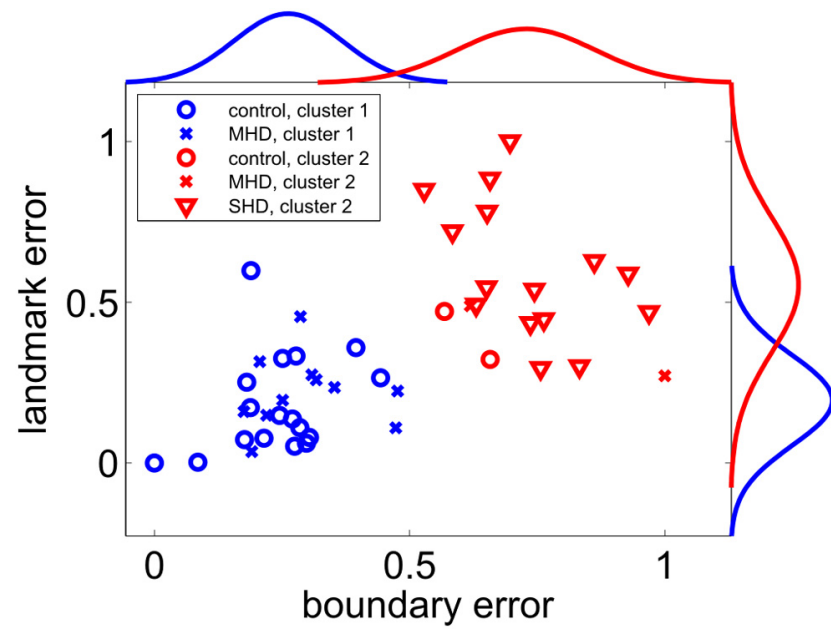

Figure 3. K-means cluster analysis separates patients with SHD from controls and from patients with MHD. Boundary errors, as well as landmark errors, were normalized across groups between 0 and 1. Two clusters were separable: one consisting mainly of patients with SHD and the other consisting exclusively of controls and those with MHD. Top and right margins represent normal distributions fit to the resulting clusters.

Canonical correlation analysis was used to investigate the relationship between the set of brain structural volumes (hippocampus, thalamus, putamen, pallidum, nucleus accumbens, caudate nucleus), on the one hand, and the set of memory measures (boundary error, landmark error, $\mathrm{MQ}$ ), on the other. Each canonical correlation is the linear correlation between canonical variables (one for the volume measures and one for the memory measures), which in turn consist of a weighted sum of the individual variables from the respective set of measures. The weights (the canonical coefficients) are chosen such that the canonical correlation is maximized. In this analysis, boundary error and landmark error were expressed as $1 /$ (boundary error) and $1 /$ (landmark error), respectively, so that large coefficients represent better performance for all measures of memory. Individual variables were centered and scaled. Larger coefficients reflect larger contributions of individual variables to the canonical correlation. We also computed correlations between the individual variables and their associated canonical variables. Large correlations of an individual variable with the canonical variables, together with small canonical coefficients, indicate that this particular variable does not contribute substantially to the canonical correlation but is correlated with the variables that do drive the canonical correlation.

\section{Navigation efficiency}

To assess participants' capacity for navigating the virtual environment in the absence of mnemonic task demand, we calculated the distance traveled in the virtual environment in the first learning block, in which objects were visible. Path lengths for all four objects were then divided by the respective optimal path lengths (the length of a straight line between the starting location and the object's position), and averaged separately for each participant. A one-way ANOVA was performed to test whether path lengths differed among experimental groups.

\section{Results}

We first used a completely data-driven approach to test whether spatial memory performance separates the three groups (SHD, $\mathrm{MHD}$, and normal controls). K-means cluster analysis, an unsupervised clustering technique, was performed using boundary and landmark errors of each participant as features. Two separable clusters emerged from these behavioral data, which corresponded to high and low boundary/landmark errors, respectively (Fig. 3). All patients with SHD fell into the high error cluster as did 2 control participants and 2 patients in the MHD group. The 
Table 1. Linear regression analysis on boundary and landmark errors using behavioral and demographic predictors

\begin{tabular}{lrr}
\hline & $F_{(1,41)}$ & $p$ \\
\hline Boundary error & & \\
Age & 0.865 & 0.358 \\
IQ & 0.133 & 0.717 \\
MQ & 22.449 & $<0.001$ \\
Sex & 4.255 & 0.046 \\
Landmark error & & \\
Age & 0.493 & 0.486 \\
IQ & 3.942 & 0.054 \\
MQ & 28.755 & $<0.001$ \\
Sex & 1.301 & 0.261 \\
\hline
\end{tabular}

remaining 17 controls and 11 patients with MHD were categorized in the low error cluster. Thus, $100 \%$ of the patients with SHD were assigned to the high error cluster, whereas $87.5 \%$ of the combined control and MHD participants were assigned to the low error cluster.

Interestingly, identical results were obtained when only the boundary error was used for clustering: The same two control participants and the same two patients with MHD fell into the high boundary error cluster, and no patient with SHD fell into the low boundary error cluster. When only the landmark error was used for clustering, two controls and two patients with MHD fell into the high landmark error cluster, and two patients with SHD, into the low landmark error cluster. Thus, the boundary error was slightly better than the landmark error in separating patients with SHD from the other two groups, and using both the landmark and boundary errors did not improve group separation as assessed by the misclassification rate.

The cluster analysis suggested that spatial memory performance of the control and MHD groups did not differ. To test this outcome more directly, we conducted two separate one-way ANOVAs using experimental group (control vs MHD) as a predictor, and boundary error and landmark error as the respective dependent variables. Neither boundary error $\left(F_{(1,30)}=2.02, p=\right.$ $0.166)$ nor landmark error $\left(F_{(1,30)}=0.59, p=0.447\right)$ differentiated the group with MHD from the control group.

We next examined the degree to which spatial memory performance could be predicted from measures of cognitive performance (IQ and MQ) as well as from demographic factors (i.e., age and sex). Two multiple regression analyses were performed, with boundary error and landmark error serving as the respective dependent variables, and IQ, MQ, age, and sex as predictor variables. As shown in Table 1, the most prominent predictor for both boundary and landmark error was MQ. A significant effect was observed for sex on boundary error (female $>$ male) and a marginal effect for IQ on landmark error (high $>$ low). Thus, both measures of spatial memory were related most closely to MQ.

To further assess the relationship between MQ and spatial memory performance, we conducted correlation analyses, across experimental groups, between boundary errors and landmark errors on the one hand, and the MQ subtests (visual immediate, visual delayed, verbal immediate, verbal delayed). All correlations were negative $(-0.68<r<-0.49)$, all with $p$ values $<0.001$. Thus, spatial memory measures correlated significantly with all MQ subtests. Furthermore, separate ANOVAs on the MQ subtests with experimental group as the predictor variable revealed highly significant group differences for all four MQ subtests $\left(\right.$ all $F_{(2,43)}>23$, all $\left.p<0.001\right)$.
Table 2. Linear regression analysis on boundary and landmark errors using brain structure volumes as predictors

\begin{tabular}{lrr}
\hline & $F_{(1,37)}$ & \multicolumn{1}{c}{$p$} \\
\hline Boundary error & & \\
$\quad$ Hippocampus & 20.236 & $<0.001$ \\
Thalamus & 0.561 & 0.459 \\
Putamen & 1.280 & 0.265 \\
Pallidum & 2.496 & 0.123 \\
Nucleus accumbens & 0.001 & 0.979 \\
$\quad$ Caudate nucleus & 1.181 & 0.284 \\
Landmark error & & \\
$\quad$ Hippocampus & 16.610 & $<.001$ \\
Thalamus & 0.434 & 0.514 \\
Putamen & 0.144 & 0.706 \\
Pallidum & 0.064 & 0.801 \\
Nucleus accumbens & 0.010 & 0.922 \\
Caudate nucleus & 0.006 & 0.941 \\
\hline
\end{tabular}

This result raises the possibility that the deficit in spatial memory is due to a general decline in memory function in patients with SHD, rather than being an indication of a separable deficit in spatial memory per se. To test whether spatial memory scores differ as a function of experimental group beyond the difference to be expected based on MQ, we conducted ANCOVAs on the boundary and landmark errors using the fixed factor of "group" as a predictor, together with MQ, IQ, age, and sex as covariates. These analyses revealed a significant effect of group for both boundary-based $\left(F_{(2,39)}=8.93, p<0.001\right)$ and landmark-based $\left(F_{(2,39)}=5.16, p=0.01\right)$ navigation, indicating that the spatial memory deficit associated with SHD is not fully captured by MQ.

To further assess whether factors other than MQ may influence spatial memory function, we conducted two analyses. First, we asked whether working memory, as assessed by the Working Memory Index (a subtest of Full Scale IQ), correlated with spatial memory measures. Working Memory Index did not correlate significantly with either boundary error $(p=0.065)$, or landmark error $(p=0.435)$. Second, we asked whether difficulties in using the keyboard to navigate the virtual environment, regardless of the memory demand of the task, may account for the apparent deficit in navigational memory. We found that the distance traveled to pick up visible objects in the very first learning block did not differ significantly among experimental groups $\left(F_{(2,44)}=\right.$ $1.06, p=0.35)$. Thus, these results suggest that neither working memory impairments nor executive function deficits, as assessed by participants' capacity to navigate to a target location in the virtual environment, are likely to be responsible for the impairment in spatial memory.

The critical question in the present experiment was whether boundary-based and landmark-based navigation rely differentially on the hippocampus and the caudate nucleus, respectively. To investigate the relationship between spatial memory performance and volumes of specific brain structures, we conducted multiple regression analyses on the boundary and landmark errors, with the following volumes as predictor variables: hippocampus, thalamus, putamen, globus pallidus, nucleus accumbens, and caudate nucleus. In this analysis, only the hippocampal volume was significantly related to either measure of spatial memory (Table 2). Furthermore, we obtained volumetric measures of parahippocampal cortex from 7 controls and from 9 patients with SHD. Regression analysis on the spatial memory measures with the hippocampus and parahippocampal cortex volumes as predictors revealed no effect of parahippocampal cortex volume on either boundary errors $\left(t_{(13)}=-0.12, p=0.91\right)$, 
or landmark errors $\left(t_{(13)}=0.09, p=\right.$ 0.92), whereas the hippocampal volumes remained highly significant. Thus, the results suggest that the hippocampus is critical for both boundary-based as well as landmark-based spatial navigation and that the caudate nucleus does not contribute significantly to either measure of spatial recall.

The MHD patients were, on average, considerably younger than the other two groups. In turn, all MHD patients received the CMS to measure MQ, whereas participants in the other two groups received either the CMS or the Wechsler's Memory Scale, Ed 3 (see Materials and Methods). Although we did not observe an effect of age on spatial memory performance (Table 1), the use of different MQ measures raises the possibility that the volumetric results were influenced by the different age distributions of the experimental groups. To address this possibility, we performed the regression analysis using the volumetric measures on the subset of participants receiving the CMS (SHD: $n=5$; MHD: $n=13$; CON: $n=12$ ). In this subgroup, the three experimental groups did not differ significantly in age $\left(F_{(2,27)}=\right.$ $0.22, p=0.805)$. Regression analysis revealed that, again, only the hippocampal volume emerged as a significant predictor for either boundary-based $\left(F_{(1,22)}=7.98, p=0.010\right)$ or landmark-based $\left(F_{(1,22)}=17.80, p<0.001\right)$ navigation. This result, together with the finding that age was not a significant predictor for spatial memory performance, suggests that the younger age of the MHD group did not substantially influence the overall finding that the hippocampal volumes, but not the volumes of other structures, predict the degree of spatial memory performance.

To further investigate the relationship between the set of volume measures (hippocampus, thalamus, putamen, pallidum, nucleus accumbens, caudate nucleus) and the set of memory measures (boundary error, landmark error, MQ), we conducted a canonical correlation analysis (see Materials and Methods). One canonical correlation was significant $(r=$ $\left.0.846, F_{(18,99.48)}=3.625, p<0.001\right)$. Figure 4 shows, for each of the volume measures and each of the memory measures, the corresponding canonical coefficients (top). The results indicate that the strongest relationship emerges between the hippocampal volume on the one hand and the MQ and boundary error on the other. The fact that the canonical coefficients for all volumes other than the hippocampus are small indicates that these volumes contribute considerably less to the canonical correlation. However, despite their small canonical coefficients, both the thalamus and the caudate nucleus showed a substantial positive correlation with the canonical variable (Fig. 4, bottom). This suggests that both are correlated with hippocampal volumes and, by extension, with the memory measures. Similarly, landmark error correlates substantially with the canonical variable, suggesting a strong relationship with boundary error and MQ and, by extension, the volume measures. However, the canonical coefficient for the landmark error is considerably smaller than those for the boundary error and $\mathrm{MQ}$, indicating that its relationship to the volume measures is largely captured by the latter two measures. To- gether, the canonical correlation analysis indicates that the hippocampus is the main predictor for all the types of memory assessed in this study and that the thalamus and caudate nucleus are related to memory function, but largely in so far as they covary with the hippocampus.

Finally, we tested whether the learning rate differed among the three groups during the four initial blocks of learning trials in which both boundary and landmark were present. A mixedeffects ANOVA on the learning errors with the factors group (fixed factor, three levels), block (continuous variable, 1-4 referring to learning blocks 2-5), and participant (random factor, nested in group), showed the following: (1) learning rate differed between groups (significant interaction between group and block: $\left.F_{(2,138)}=3.48, p=0.034\right)$; (2) overall performance differed between groups (significant main effect of group: $F_{(2,177.6)}$ $=4.56, p=0.012$ ); and (3) performance increased as a function of block (significant main effect of block $F_{(1,138)}=14.66, p<$ $0.001)$. Post hoc analyses on the three groups individually revealed that whereas both the control participants $\left(F_{(1,56)}=14.14, p<\right.$ $0.001)$ and the patients with $\operatorname{MHD}\left(F_{(1,38)}=20.63, p<0.001\right)$ improved significantly across the four blocks, the patients with SHD did not $\left(F_{(1,44)}=0.005, p=0.943\right)$. The learning data are displayed in Figure 5.

\section{Discussion}

The present study sheds light on the nature of the impairment in spatial memory recall in patients with hippocampal damage. Compared with healthy controls and patients with MHD (volume reduction mean, $19.3 \%$; range, $14.6 \%-25.8 \%$ ), patients with SHD (volume reduction mean, $46.6 \%$; range, 30.4\%-66.8\%) were markedly impaired in the recall of object-location information in a virtual environment. This impairment was apparent both when navigation relied on boundaries surrounding the environment, as well as when it relied on a landmark as a local spatial reference. Furthermore, patients with SHD did not improve significantly in spatial recall accuracy during four initial learning trials when both boundary and landmark were present. Finally, the results indicate that navigational allocentric spatial 


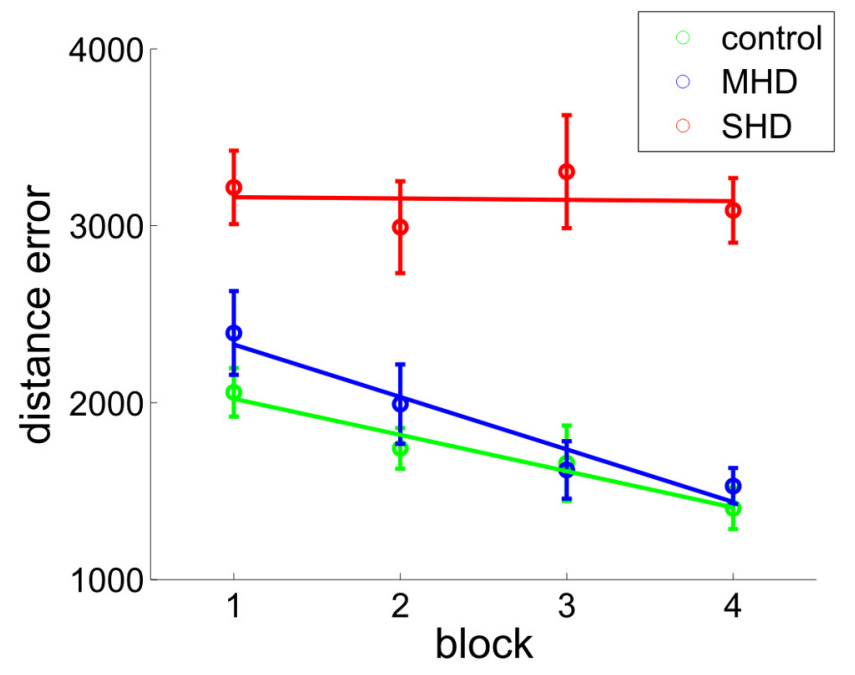

Figure 5. Learning data. Patients with MHD and control participants improve recall performance (i.e., lower the distance error) as a function of block. Patients with SHD exhibit poorer recall performance and do not improve as a function of block.

memory function is not fully accounted for by MQ, a widely used measure of general memory function.

In patients who suffered hypoxic/ischemic events early in life, the hippocampus is the structure that exhibits the greatest and most consistent atrophy. Hence, memory impairments in those patients have been largely attributed to a loss of hippocampal function. Although the multiple regression analysis in the present study indicated that hippocampal volume reduction, but not volume reduction of other structures, explains memory impairment in our spatial recall task, the present data do not allow unequivocal conclusions as to whether extra-hippocampal volume reductions are at least partly responsible for the behavioral deficit. However, studies in experimental animals using lesions or inactivations of the hippocampus in the absence of damage to those other structures show that hippocampal damage alone leads to impairments in allocentric spatial memory (Morris et al., 1982, 1986; Packard and McGaugh, 1996; Pearce et al., 1998; Hampton et al., 2004; Lavenex et al., 2006; Glavis-Bloom et al., 2013; Forcelli et al., 2014). In light of those experimental findings, our patient data support the view that the hippocampus is a key structure for spatial memory function in humans as well and that hippocampal atrophy is the most likely cause of the spatial memory deficits we observed.

Contrary to the SHD group, the MHD group in the present study did not show significantly impaired spatial recall performance. This group of patients was, on average, younger than both the control and SHD groups. However, across groups, age did not predict spatial memory performance, and regression analysis on a subgroup of young participants (who received the Children's Memory Scale) showed qualitatively highly similar results to the analysis including all participants, in that hippocampal volume strongly predicted spatial recall performance. By extension, the results suggest that the hippocampal volume reduction in the MHD group was insufficient to produce a reliable impairment in spatial recall but provide no evidence in support of the notion that patients with MHD were unimpaired because of their lower average age.

A number of studies in single patients with relatively selective hippocampal damage (Holdstock et al., 2000; Spiers et al., 2001; King et al., 2002, 2004; Hartley et al., 2007) have revealed deficits in allocentric spatial memory. At the same time, it has been pro- posed that the reliance of allocentric memory on the hippocampus may not be absolute, in that allocentric spatial information can be learned not only via a medial temporal cognitive memory system, but also via a dorsal striatal habit system. In a series of behavioral experiments in humans, Doeller and Burgess (2008) showed that learning of allocentric spatial information in a virtual environment occurs incidentally in relation to local boundaries but follows associative reinforcement principles (exhibiting blocking and overshadowing) when learning occurs in relation to local landmarks. In an fMRI experiment using a similar task, it was shown that learning and remembering object locations relative to local boundaries were associated with activity in the right posterior hippocampus, whereas learning and remembering object locations relative to local landmarks were associated with activity in the right dorsal striatum (Doeller et al., 2008). An intuitive hypothesis derived from those studies would thus predict that patients with selective hippocampal damage are more impaired in boundary-based than in landmark-based spatial navigation. The present data, however, suggest that patients with SHD are similarly impaired in both types of navigational learning. There are several different possibilities that could have led to this result.

First, it may be argued that subtle damage to the dorsal striatum alone is indeed responsible for the substantial deficit in landmark-based navigational memory. However, in light of the considerably more pronounced volume reduction of the hippocampus than of the caudate nucleus, together with comparable impairments in the boundary and landmark conditions that were both best explained by hippocampal volume reductions, we do not consider it likely that minor damage to the caudate nucleus was solely responsible for the substantial deficit in landmarkbased navigation.

An alternative hypothesis may be that there is a differential dependence of boundary- and landmark-based navigation on the hippocampus and the dorsal striatum, respectively, but that the dissociation is not absolute. Although both boundary- and landmark-based navigation rely on hippocampal integrity, landmark navigation could rely in addition on the dorsal striatum. If this were the case, one might expect the behavioral pattern observed in the present study in patients with hippocampal damage, whereas patients with additional dorsal striatal damage may be disproportionately impaired in landmark-based navigation, thus raising an interesting question for future research. Furthermore, both boundary- and landmark-based navigation may rely on the hippocampus as well as on the dorsal striatum, with differences being a matter of magnitude, rather than exclusivity. Such differences in degree may well explain differential patterns of activation in fMRI (Doeller et al., 2008).

In addition, a differential involvement of the hippocampus and the dorsal striatum for boundary- and landmark-based navigation may be one not of degree, but of timing. Although Doeller and colleagues (Doeller and Burgess, 2008; Doeller et al., 2008) did not find pronounced differences in the time courses of learning object locations relative to boundaries and landmarks, evidence from experimental animals suggests that striataldependent habit learning tends to control behavior later during learning than medial-temporal-dependent cognitive learning when both systems are intact (Packard and McGaugh, 1996). Thus, it is possible that a striatal system capable of subserving landmark-based navigation is relatively intact in patients with SHD but requires an intact hippocampal system early in training to facilitate the formation of a viable striatal representation. 
Little is known about whether and how neural systems are reorganized in response to hypoxic/ischemic events leading to bilateral hippocampal atrophy. However, memory impairments are relatively consistently expressed after bilateral hippocampal damage sustained between birth and puberty (Vargha-Khadem et al., 2003), a period during which the potential for compensation is at its highest. This suggests that it is the nature of the damage that leads to behavioral deficits, and that compensatory mechanisms cannot rescue behavioral performance, at least as long as the damage is bilateral. However, the present data do not exclude the possibility that boundary- and landmark-based navigation rely differentially on the hippocampus and the dorsal striatum in healthy controls, but that neural plasticity in patients with SHD may have led to structural and/or functional reorganization that prevented a striatal system for landmark navigation from developing normally. Further studies with different patient populations, as well as comparative studies in experimental animals, are needed to shed light on this question.

Yet another explanation for an absence of a dissociation between boundary- and landmark- based navigation in the present study is that patients with SHD may adopt strategies that are different from the ones used by control participants. Because of the subjective difficulty of the memory task, patients may exhibit guessing behavior as an active strategy for both boundary- and landmark-based navigation. Thus, patients may decide to drop the object anywhere because the location is not known, thereby overwriting the possible use of a striatal habit-based system that may otherwise be able to subserve landmark-based navigation (similar to the potential need for early hippocampal learning discussed above). Interestingly, in a study investigating face and scene memory in the patient Jon, the authors discussed the possibility that the differential pattern of receiver operating characteristic curves between tasks may be an idiosyncratic result of coping with low memory capacity (Bird et al., 2008). If patients with SHD as a group adopt strategies that are less optimal than the strategies of control participants, and if the same strategy is employed for both landmark- and boundary-based navigation, then this too could have led to the present result (see also Schuck et al., 2013).

The existence of dissociable striatal habit (Mishkin et al., 1984; Knowlton et al., 1996; Fernandez-Ruiz et al., 2001; Bayley et al., 2005; Yin and Knowlton, 2006) and medial temporal cognitive memory systems (O'Keefe and Nadel, 1978; Eichenbaum et al., 1996; Mishkin et al., 1997; Squire and Wixted, 2011) is widely agreed upon. Thus, perhaps the most likely explanation for the present pattern of results is that both boundary- and landmarkbased navigation, as employed in the present experiment, do indeed depend on hippocampal integrity. One possible explanation for this finding is that participants started successive trials from a different starting position, which may prevent habitual stimulusresponse behavior based on heading vectors from developing (Pearce et al., 1998). Furthermore, participants were instructed before the experiment to avoid first moving toward the same location on each trial and then finding the target location from this same position on each trial. Thus, participants may have treated both the boundary as well as the landmark condition as one that is to be solved according to allocentric spatial relational principles that in turn depend on hippocampal integrity.

Together, the present results show that patients with SHD are impaired in learning and recalling allocentric spatial information in a virtual environment, that this impairment is best explained by hippocampal volume reduction, and that this damage impairs allocentric navigation based on boundaries and local landmarks similarly and beyond what can be predicted by MQ as a widely used measure of general memory function. Thus, in addition to clarifying the role of the human hippocampus in allocentric navigational memory, the results suggest that MQ does not capture all aspects of memory dysfunction in patients with relatively selective hippocampal volume reduction due to hypoxia/ischemia.

\section{References}

Adlam AL, Malloy M, Mishkin M, Vargha-Khadem F (2009) Dissociation between recognition and recall in developmental amnesia. Neuropsychologia 47:2207-2210. CrossRef Medline

Bayley PJ, Frascino JC, Squire LR (2005) Robust habit learning in the absence of awareness and independent of the medial temporal lobe. Nature 436:550-553. CrossRef Medline

Bird CM, Vargha-Khadem F, Burgess N (2008) Impaired memory for scenes but not faces in developmental hippocampal amnesia: a case study. Neuropsychologia 46:1050-1059. CrossRef Medline

Caliński T, Harabasz J (1974) A dendrite method for cluster analysis. Commun Stat 3:1-27. CrossRef

Cohen M (1997) Children's Memory Scale. San Antonio, TX: Harcourt Brace and Co.

Cooper JM, Gadian DG, Jentschke S, Goldman A, Munoz M, Pitts G, Banks T, Chong WK, Hoskote A, Deanfield J, Baldeweg T, de Haan M, Mishkin M, Vargha-Khadem F (2015) Neonatal hypoxia, hippocampal atrophy, and memory impairment: evidence of a causal sequence. Cereb Cortex 25: 1469-1476. CrossRef Medline

Doeller CF, Burgess N (2008) Distinct error-correcting and incidental learning of location relative to landmarks and boundaries. Proc Natl Acad Sci U S A 105:5909-5914. CrossRef Medline

Doeller CF, King JA, Burgess N (2008) Parallel striatal and hippocampal systems for landmarks and boundaries in spatial memory. Proc Natl Acad Sci U S A 105:5915-5920. CrossRef Medline

Eichenbaum H, Schoenbaum G, Young B, Bunsey M (1996) Functional organization of the hippocampal memory system. Proc Natl Acad Sci U S A 93:13500-13507. CrossRef Medline

Fernandez-Ruiz J, Wang J, Aigner TG, Mishkin M (2001) Visual habit formation in monkeys with neurotoxic lesions of the ventrocaudal neostriatum. Proc Natl Acad Sci U S A 98:4196-4201. CrossRef Medline

Forcelli PA, Palchik G, Leath T, DesJardin JT, Gale K, Malkova L (2014) Memory loss in a nonnavigational spatial task after hippocampal inactivation in monkeys. Proc Natl Acad Sci U S A 111:4315-4320. CrossRef Medline

Glavis-Bloom C, Alvarado MC, Bachevalier J (2013) Neonatal hippocampal damage impairs specific food/place associations in adult macaques. Behav Neurosci 127:9-22. CrossRef Medline

Hampton RR, Hampstead BM, Murray EA (2004) Selective hippocampal damage in rhesus monkeys impairs spatial memory in an open-field test. Hippocampus 14:808-818. CrossRef Medline

Hartley T, Bird CM, Chan D, Cipolotti L, Husain M, Vargha-Khadem F, Burgess N (2007) The hippocampus is required for short-term topographical memory in humans. Hippocampus 17:34-48. CrossRef Medline

Holdstock JS, Mayes AR, Cezayirli E, Isaac CL, Aggleton JP, Roberts N (2000) A comparison of egocentric and allocentric spatial memory in a patient with selective hippocampal damage. Neuropsychologia 38:410425. CrossRef Medline

Isaacs EB, Lucas A, Chong WK, Wood SJ, Johnson CL, Marshall C, VarghaKhadem F, Gadian DG (2000) Hippocampal volume and everyday memory in children of very low birth weight. Pediatr Res 47:713-720. CrossRef Medline

Isaacs EB, Vargha-Khadem F, Watkins KE, Lucas A, Mishkin M, Gadian DG (2003) Developmental amnesia and its relationship to degree of hippocampal atrophy. Proc Natl Acad Sci U S A 100:13060-13063. CrossRef Medline

King JA, Burgess N, Hartley T, Vargha-Khadem F, O’Keefe J (2002) Human hippocampus and viewpoint dependence in spatial memory. Hippocampus 12:811-820. CrossRef Medline

King JA, Trinkler I, Hartley T, Vargha-Khadem F, Burgess N (2004) The hippocampal role in spatial memory and the familiarity-recollection distinction: a case study. Neuropsychology 18:405-417. CrossRef Medline

Knowlton BJ, Mangels JA, Squire LR (1996) A neostriatal habit learning system in humans. Science 273:1399-1402. CrossRef Medline 
Kumaran D, Hassabis D, Spiers HJ, Vann SD, Vargha-Khadem F, Maguire EA (2007) Impaired spatial and non-spatial configural learning in patients with hippocampal pathology. Neuropsychologia 45:2699-2711. CrossRef Medline

Lavenex PB, Amaral DG, Lavenex P (2006) Hippocampal lesion prevents spatial relational learning in adult macaque monkeys. J Neurosci 26: 4546-4558. CrossRef Medline

Mishkin M, Malamut B, Bachevalier J (1984) Memories and habits: two neural systems. In: Neurobiology of learning and memory (Lynch G, McGaugh JL, Weinberger NM, eds), pp 65-77. New York: Guilford.

Mishkin M, Suzuki WA, Gadian DG, Vargha-Khadem F (1997) Hierarchical organization of cognitive memory. Philos Trans R Soc Lond B Biol Sci 352:1461-1467. CrossRef Medline

Morris RG, Garrud P, Rawlins JN, O'Keefe J (1982) Place navigation impaired in rats with hippocampal lesions. Nature 297:681-683. CrossRef Medline

Morris RG, Hagan JJ, Rawlins JN (1986) Allocentric spatial learning by hippocampectomised rats: a further test of the "spatial mapping" and "working memory" theories of hippocampal function. Q J Exp Psychol B 38: 365-395. CrossRef Medline

O’Keefe J, Nadel L (1978) The hippocampus as a cognitive map. Oxford: Oxford UP.

Packard MG, McGaugh JL (1996) Inactivation of hippocampus or caudate nucleus with lidocaine differentially affects expression of place and response learning. Neurobiol Learn Mem 65:65-72. CrossRef Medline

Patenaude B, Smith SM, Kennedy DN, Jenkinson M (2011) A Bayesian model of shape and appearance for subcortical brain segmentation. Neuroimage 56:907-922. CrossRef Medline
Pearce JM, Roberts AD, Good M (1998) Hippocampal lesions disrupt navigation based on cognitive maps but not heading vectors. Nature 396: 75-77. CrossRef Medline

Schuck NW, Doeller CF, Schjeide BM, Schröder J, Frensch PA, Bertram L, Li SC (2013) Aging and KIBRA/WWC1 genotype affect spatial memory processes in a virtual navigation task. Hippocampus 23:919-930. CrossRef Medline

Spiers HJ, Burgess N, Hartley T, Vargha-Khadem F, O'Keefe J (2001) Bilateral hippocampal pathology impairs topographical and episodic memory but not visual pattern matching. Hippocampus 11:715-725. CrossRef Medline

Squire LR, Wixted JT (2011) The cognitive neuroscience of human memory since H.M. Annu Rev Neurosci 34:259-288. CrossRef Medline

Vargha-Khadem F, Gadian DG, Watkins KE, Connelly A, Van Paesschen W, Mishkin M (1997) Differential effects of early hippocampal pathology on episodic and semantic memory. Science 277:376-380. CrossRef Medline

Vargha-Khadem F, Salmond CH, Watkins KE, Friston KJ, Gadian DG, Mishkin M (2003) Developmental amnesia: effect of age at injury. Proc Natl Acad Sci U S A 100:10055-10060. CrossRef Medline

Wechsler D (1997) Wechsler Adult Intelligence Scale, Ed 3. London: The Psychological Corporation.

Wechsler D (2003) Wechsler Intelligence Scale for Children, Ed 4, administration and scoring manual. San Antonio, TX: The Psychological Corporation.

Yin HH, Knowlton BJ (2006) The role of the basal ganglia in habit formation. Nat Rev Neurosci 7:464-476. CrossRef Medline 